\title{
Microfluidics in Single Cell Analysis
}

\author{
Caroline Beck and Mattias Goksör \\ University of Gothenburg \\ Sweden
}

\section{Introduction}

Microfluidics is a research field that evolved during the early 1980's and is still under constant and rapid progress. As indicated by the name, microfluidics concerns the behaviour of fluids in micro length scale environments and originally has its roots in four different disciplines: molecular biology and analysis, microelectronics and, maybe a bit surprisingly, biodefence (Whitesides 2006). The applications of microfluidic systems are usually called $\mu$ TAS (micro total analysis systems) or LOC (lab-on-a-chip) devices, and refer to the possibility to scale down analyses and reactions to a miniaturized format, something that has opened up new exciting applications. Analytical systems for DNA sequencing, separation and manipulation, enzymatic assays, protein analysis, cell sorting, counting and culturing etc. are applications readily applied (Beebe \& Paguirigan 2008; Jensen et al 2006; Manz et al 2002a; Manz et al 2002b; Tudos et al 2001; van den Berg \& Andersson 2003; van den Berg \& Lammerink 1998; Weibel et al 2007). Even in the fast growing research field of systems biology, microfluidic systems have come to play an important role. Systems biology aims to reduce and describe complex biological systems with mechanistic models such as chemical kinetics and control theory. The models are in turn used to simulate and predict the behaviour of a biological system (Kitano 2002). In order to refine these models, traditional population based data from e.g. Western blot and microarrays, needs to be complemented with data acquired from single cells (Ryley \& Pereira-Smith 2006). Since a microfluidic device offers the possibility to spatially and temporally control the fluid within, it has proven to be a powerful tool when following biological responses to perturbations on the level of individual cells (Bennett et al 2008). In traditional methodology, the removal of a specific substance or drug from cells to investigate a certain response is, if not impossible, difficult without changing the equilibrium of the whole cell culture. It is also possible that, depending on the method of investigation, the time required to change the cellular environment actually takes longer than the event you are trying to capture. Hence, the temporal resolution of such an experiment would be less than required and likely impossible to improve by traditional methods. However, these obstacles may be overcome by the use of microfluidics. Other benefits of microfluidics are vastly reduced sample and reagent volumes, lower costs and power consumption and the design versatility (Whitesides \& Sia 2003). 


\section{Single cell analysis}

Basic biological processes are often studied using a reductionist approach. Thus, only a few isolated events are monitored and any interference from external factors is kept to a minimum. The use of model organisms has gained much attraction and has contributed to the understanding of other higher species. Since many fundamental properties are conserved throughout the evolution, the investigation of simpler life forms can thus contribute to the understanding of human biological mechanisms and diseases. Within medical research, model organisms such as rats and mice designed to express a specific set of genes are often used. In molecular biology as well as in genetics, model organisms include bacteria, yeast cells, banana flies and plants. Often in vivo (within the living) experiments are separated from in vitro (in glass) experiments, where the latter indicate that the biochemical process is removed from its natural environment and instead takes place in an artificial environment. However, a cell biologist would instead use this term when referring to cells cultured outside the specific organism from which they are retrieved. In vitro experiments allow manipulations and measurements that would be impossible in vivo. In addition, an in vitro experimentalist may take advantage of the possibility to use genetically homogenous cell populations. However, also in populations with low cell-to-cell variation, traditional averaging techniques only provide the mean response for the cell population as a whole.However, also in populations with low cell-to-cell variation, traditional averaging techniques only provide the mean response for the cell population as a whole. Any information on the heterogeneity within such a cell culture, although genetically homogenous, will thus be lost. The remaining cell-to-cell variation depends on intrinsic and extrinsic noise (O'Shea \& Raser 2004) and concerns differences in the concentration of regulatory proteins or bio-molecules, cell state (cell cycle stage and cell age) as well as involved reactions and in which order these occur within the cell. These parameters play a crucial role in the biological processes and will of course mirror the experimental results.

A schematic example of the response from a cell culture measured with a bulk measurement technique that could be misinterpreted is illustrated in figure 1 and 2 . In the first example (Figure 1) the cells respond to an external stimulus either (left) as a homogenous population (all cells react at the same time and approximately to the same extent); (center) in a bimodal distribution (only a fraction of the cells react, but the reaction is to the fullest extent); or (right) the cells show a response as a combination of the two. Hence, both the kinetics and measured mean values might not correspond to the actual scenario taking place. In the second example (Figure 2) the single cell data reveal a much faster response to outer stimuli than the averaged response. Since the response is temporally distributed an averaged result will thus be falsely interpreted. Here is where single cell analysis becomes a vital and complementary tool to traditional techniques when interpreting biological events.

There are techniques available that to some extent can separate a cell population into subpopulations. FACS (Fluorescent Activated Cell Sorting) utilises fluorescence and lightscattering properties of individual cells that pass through a laser beam (Dean \& Hoffman 2007). This results in high throughput data and the cells can even be separated into subpopulations and collected for further analyses.

However, since the cells are in constant motion through the laser beam they are therefore impossible to track. Hence, there is no possibility to do time series experiments or to reanalyse a specific cell. Re-analyses of specific cells are, however, possible with the technique, laser scanning cytometry. Here, labeled cells are located on a glass slide and their emitted 
fluorescence is recorded with a microscope. This technique will give detailed morphological information but has limited possibilities to sort the cells into sub-populations. Even though the throughput is lower compared to FACS, this limitation could be overcome by a high content screening set up in which a large number of cells can be imaged over time (Deptala et al 2001). One should remember that high quality single cell analysis should be accompanied by relevant statistics. If possible, the techniques should combine environmental control with cell manipulation in an attempt to limit the extracellular influences that otherwise would broaden the population distribution. With the progressions within $\mu \mathrm{TAS} / \mathrm{LOC}$ development and automated analyses approaches, it is only a question of time before single cell analysis and high throughput studies will be established (Pepperkok \& Ellenberg 2006).
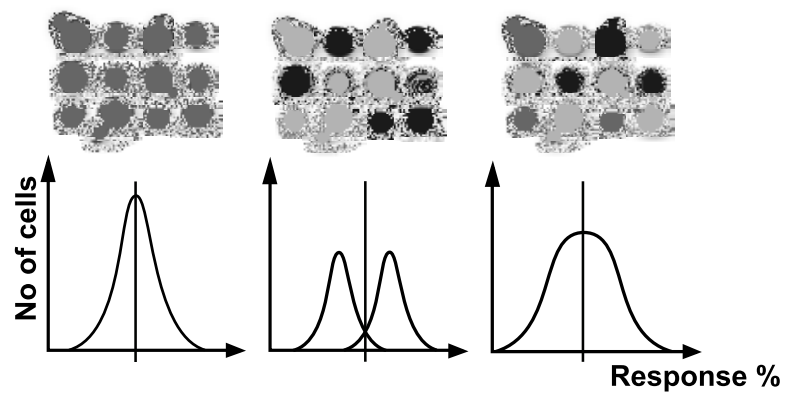

Fig. 1. Heterogenous responses may be obscured in population techniques. Population data of cells represents the mean response value from all cells studied. Hence, it is impossible to distinguish from the mean response value if the cells within the population react in the same way; as a homogenous population where all cells react at the same time and approximately to the same extent (left); in a bimodal distribution where only a fraction of the cells react to the fullest (centre); or the cells show a response as a combination of the two (right). The mean values correspond to the vertical black lines.

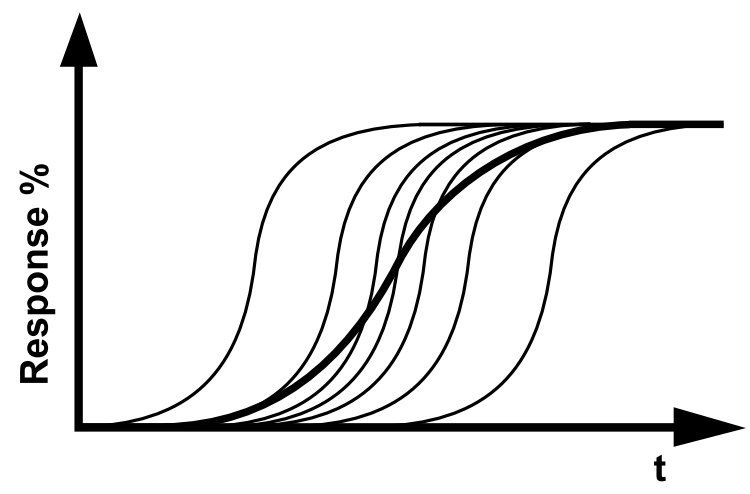

Fig. 2. Differences in measured response time of single cells versus population. Averaging techniques could be misleading when interpreting the cellular kinetic response. The mean response value of the population (thick line) shows a slower response over time $(t)$ than the individual cell responses (thin lines). 


\subsection{Yeast cells as model organism}

Saccharomyces cerevisiae (S. cerevisiae) is often known in popular parlance as baker's yeast or budding yeast, and belongs to the fungi species. The organism commonly inhabits sugar containing substrates (fruits and flowers) and can adapt to a wide range of environmental conditions. It can tolerate temperatures between freezing and $55^{\circ} \mathrm{C}$ (although proliferation occurs between 12 and $40^{\circ} \mathrm{C}$ ), grows in both acidic and moderately alkaline milieus ( $\mathrm{pH} 2.8$ $8.0)$ and tolerates both high sucrose $(3 \mathrm{M})$ and ethanol $(20 \%)$ concentrations. Not surprisingly, S. cerevisiae is the main organism used in both wine and beer production, but is also used for drug screening and functional analyses as well as a safe production organism of commercially important proteins (Bayne et al 1988; Mcaleer et al 1984). Many eukaryotic characteristics were first discovered in yeast and due to powerful genetics and molecular biology, yeast is today used not only to study the function of living cells, but also to improve or generate new biotechnological approaches (Stagljar et al 2006). S. cerevisiae is a unicellular organism, approximately $4-7 \mu \mathrm{m}$ in size, and represents the simplest form of eukaryotic cell, i.e. complex internal structures are enclosed by membranes. Several processes, such as chromosome replication and cell cycle control, intracellular signal transductions, as well as transcription and translation events, are similar between $S$. cerevisiae and higher eukaryotic cells and therefore frequently studied in yeast. However, S. cerevisiae is protected from the extracellular milieu by a cell wall (Klis et al 2006), a feature distinguishing them from mammalian cells but shared with bacteria, algae and plants. In 1996 the complete genome of S. cerevisiae was successfully sequenced (Goffeau et al 1996) and the 16 linear chromosomes were shown to consist of approximately 13 million base pairs (including the ribosomal DNA) encoding in total roughly 6000 open reading frames (ORFs). Even though it was the first eukaryote genome ever sequenced, molecular geneticists had transformed S. cerevisiae with foreign DNA already in 1978 (Beggs 1978; Hinnen et al 1978). Nearly 31\% of the possible ORFs show statistically robust homologues in the mammalian genomes and many of the known genes in S. cerevisiae have mammalian orthologs (Ploger et al 2000). This makes yeast an ideal model organism in which biological events that could have future impact on disease prevention, easily can be studied (Hartwell 2004). The knowledge of yeast genetics is today vast and summarised at the public Saccharomyces Genome Database (www.yeastgenome.org), where various search options and analysis tools for homology and gene comparisons are also available. The knowledge of each gene function is a joint goal for the yeast community. This is facilitated by the yeast GFP clone collection, containing approximately $75 \%$ of all yeast genes tagged to green fluorescent protein (GFP), enabling protein detection and localization (http:// http://yeastgfp.yeastgenome.org/). Yeast is overall a very robust model organism to work with; it is easy to cultivate, has a short generation time (about 90 minute), is non-pathogenic and possesses a well known genome that easily can be manipulated. S. cerevisiae are grown on solid agar plates or in liquid nutrient broth and can survive storage in glycerol stocks at $-70^{\circ} \mathrm{C}$ for long periods of time. The most common liquid nutrient is yeast peptone dextrose (YPD). However, since YPD is highly auto-fluorescent, a synthetic minimum yeast nitrogen base (YNB) medium is used for fluorescent imaging applications.

In nature $S$. cerevisiae is found as diploids, meaning that they have two copies of their chromosomes, probably since the extra gene copy increases their chances of surviving essential gene mutations. They divide by budding off new cells (hence the name budding yeast) which results in two genetically identical cells of unequal size, a larger one (the 
mother) and a smaller one (the daughter). After budding, every daughter cell leaves a bud scar on the cell wall of the mother cell. Mother cells may bud as many as 30-50 times and hence have the same amount of bud scars (Mortimer \& Johnston 1959). Under certain circumstances, such as e.g. nitrogen starvation, the diploid cells sporulate and form four haploid (one copy of each chromosome) spores, of which usually only a single spore survives. This spore will in turn germinate when the nutrient access is sufficient and reproduce as a haploid cell. Two haploid cells can via a sexual phase, however, fuse to a diploid cell, provided they express the opposite mating types, a and a (Burgess 2003). This mating can even happen between mother and daughter cells, since the mother cell can switch mating type. The life cycle of $S$. cerevisiae has many advantages. The spores can survive harsh environments and the sporulation event "cleans" the genome from any possible accumulated mutations and gives rise to genetic diversity by allele crossover. Furthermore, mating partners from other spores might result in possible genetic advantages. In a laboratory environment the yeast cells are kept as stable haploid cell cultures genetically modified so that no mating type switches are possible.

\subsubsection{HOG - A MAPK pathway}

External perturbations of a cell are sensed and conveyed into its interior by processes referred to as signal transduction. Even though the perturbation can differ from stress conditions and growth factors to specific cytokines and hormones, the signal transduction triggers an appropriate response to counteract or adapt the cell to the new environment. The principles of the intracellular signal transductions of S. cerevisiae are considered to be well conserved, especially those belonging to the so called mitogen activated protein (MAP) kinase pathways (Shields et al 1999). These pathways all share a common architecture where the most downstream component, the MAP kinase (MAPK), becomes phosphorylated and thereby activated by its upstream MAPK kinase (MAPKK) (Figure 3). This MAPKK is in turn phosphorylated by a further upstream MAPKK kinase (MAPKKK).

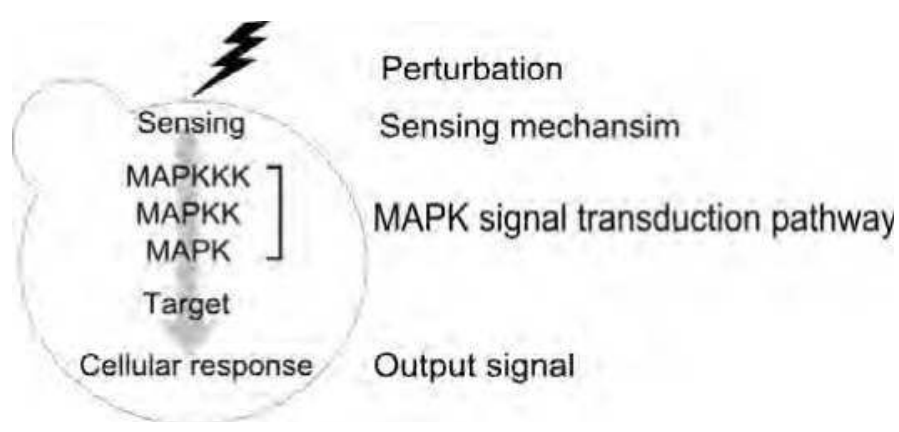

Fig. 3. The common architecture of a typical MAPK signal transduction pathway in yeast. Outer stimulus is sensed and transduced into the interior of the cell via the phosphorylation events of the pathway. When reaching the targets, the output signal corresponds to a cellular response to counteract and/or adapt the cell to the perturbation.

An external stimulus is responsible for activating this most upstream kinase of the signal transduction pathway that leads to the cellular response. The High Osmolarity Glycerol 
(HOG) response pathway is one of four MAPK pathways present in S. cerevisiae (Figure 4) (Gustin et al 1998). The perturbation is constituted by hyper osmotic stress and results in glycerol production to prevent dehydration (Thorner et al 2004). The signal transduction is mediated by activated Hog1 (Hog1-PP) which migrates from the cytosol to the cell nucleus via the nuclear importer Nmd5 (Silver et al 1998). Inside the nucleus the transcription of up to 600 genes is up regulated (Thorner et al 2004). The HOG pathway has two separate branches of activation, the $\operatorname{Sin} 1$ and the Sho1 branch. In total, three plasma transmembrane sensors activate the pathway; Sln1, Msb2 and Hkr1, where the latter two belong to the Sho1 branch (Posas et al 1996; Tatebayashi et al 2007). Together with two other components, Ypd1 and Ssk1, Sln1 constitutes what is called a phospho-relay module. $\operatorname{Sln} 1$ is active under normal osmotic conditions and becomes inactivated during hyper osmotic stress. When active, $\operatorname{Sln} 1$ is auto-phosphorylated and the phospho-group is mediated via Ypd1 to Ssk1. Ssk1 acts as a response regulator and becomes inactivated when receiving the phosphogroup, i.e. does not activate the downstream transduction components (Saito \& Posas 1998).

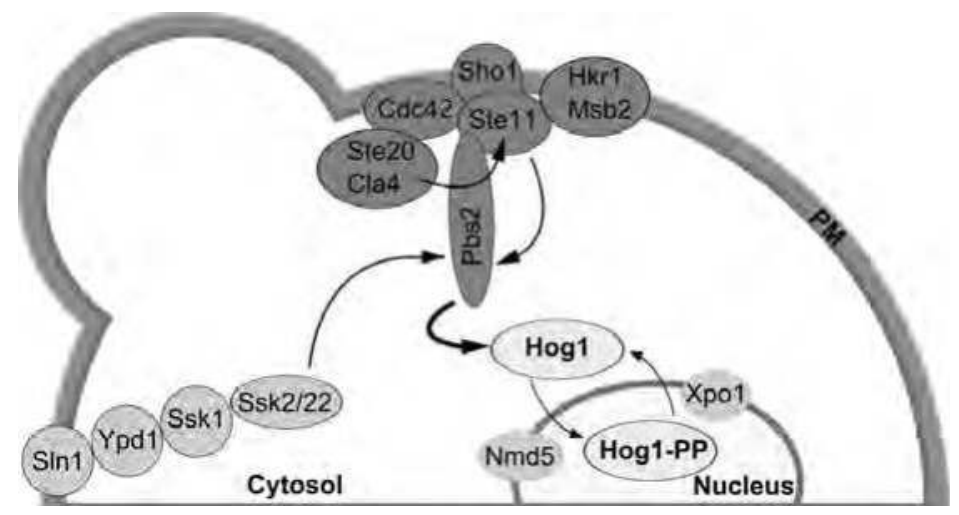

Fig. 4. A schematic overview of the HOG pathway in S. cerevisiae. The pathway sensors (Sln1, Msb2 and Hkr1) are situated in the plasma membrane (PM) of the cell. Upon high osmolarity the cytosolic MAPK Hog1 is phosphorylated (Hog1-PP) by the upstream MAPKK Pbs2. Pbs2 in turn can be activated by two separate sensing branches, the $\operatorname{Sin} 1$ and Sho1 branch. Upon activation, Hog1 migrates into the nucleus via the nuclear importer Nmd5 where it binds to specific DNA regions and together with cofactors up regulates gene transcription. Upon dephosphorylation and inactivation, Hog1 is rerouted into the cytosol via the exporter protein Xpo1.

However, during hyperosmotic stress $\operatorname{Sln} 1$ is dephosphorylated and consequently the amount of unphosphorylated Ssk1 accumulates. Ssk1 binds to the components Ssk2 and Ssk22, which becomes auto-phosphorylated and activated, and in turn can activate the MAPKK Pbs2 by yet another phosphorylation. The activation mechanism of the Sho1 branch is still partly unknown. Since Sho1 is a transmembrane protein it was first thought to act as a sensor of this branch (Maeda et al 1995). Instead, it seems to be important as a scaffold protein, recruiting other components important for active cell growth and remodeling of the cell membrane (Ammerer et al 2000). The stimulation of the Sho1 branch sensors probably recruits the MAPKK Pbs2 to the cell membrane, which will then act as a scaffold protein for the other components of the Sho1 branch. The kinases Ste11, Ste20 and Cla4 will in 
association with the G-protein Cdc42 all contribute to the phosphorylation of Pbs2 (Hohmann 2009).

As previously described, the two branches merge by the activation of the MAPKK Pbs2 that in turn phosphorylates and activates the transcription factor and MAPK, Hog1. Although functionally redundant, the $\operatorname{Sln} 1$ branch is more sensitive and shows gradual activation characteristics, while the Sho1 branch requires a higher degree of osmosis to react in an "allor-nothing" fashion (O'Rourke \& Herskowitz 2004). The cellular response to the increased osmolarity involves transcription of both Hog1 specific and general stress-dependent genes, which are thought of as the cells' long-term adaptation to the new environment (Ferreira et al 2005; Rep et al 2000; Rep et al 1999). However, since cells are still able to adapt to increased osmolarity in the absence of nuclearized Hog1 (Thorner et al 2008), mechanisms extending beyond gene transcription seem to be of higher importance when instant adaption is required (Dihazi et al 2004; Tamas et al 2006). Since a constitutively active Hog1 can be detrimental to the cell, the amplitude and period of Hog1 activation needs to be precisely controlled. This is achieved by dephosphorylation of Hog1. Several different phosphatases can act on Hog1 which allows precise temporal and spatial modulation of its activity (Molina et al 2005; Warmka et al 2001). When Hog1 is dephosphorylated it returns to the cell cytosol via the nuclear exportin, Xpo1, where it awaits a new perturbation (Silver et al 1998). The shuttling between cytosol and nucleus is thought to correspond to the activity of Hog1. The mammalian Hog1 ortholog, MAPK p38, is involved in chronic inflammatory diseases and its four isoforms are activated upon different environmental stresses such as e.g. inflammatory cytokines, hypoxia and oxidative stress (Ono \& Han 2000).

\subsubsection{Inhibitors}

The alterations of gene expression for most model organisms are today possible through molecular biology and cloning techniques. The transient transformation (or transfection) of cells with a plasmid will usually result in an over expression of the gene of interest. Mutations can also be introduced by transiently or permanently altering the gene expression by changing the genetic code. Some of these mutations will lead to non-viable changes and hence kill the cell while other mutations will not affect the cell at all. Some mutations will, however, change the targeted gene expression as intended. Unfortunately, also, other gene expressions than the one targeted can change as a secondary effect. This is referred to as compensatory mechanisms (Specht \& Shokat 2002). This "adaption mechanism" for the model organism to the new environmental parameters may interfere with the initial purpose of the cloning and consequently make the data more difficult to interpret. Compensatory mechanisms are especially common in deletion mutants, resulting in interrupted pathways, and as a consequence other intertwined pathways or similar proteins could compensate for the loss.

An alternative approach is to instead use a substance that can be administrated to the cells that will inhibit or disable a specific protein or group of proteins. This will allow studies of response dynamics without any compensatory mechanisms. Since protein kinases share a common sequence and structural homology in their ATP-binding site, many synthesized kinase inhibitors lack selectivity (Johnson et al 2004). Approaches have been conveyed where a protein inhibitor is synthesized to recognize a mutated version of the protein kinase 
of interest, referred to as the ASKA approach (Shokat et al 2000). It is, however, more convenient to use the wild type protein kinase not only in order to abolish the need of molecular genetics work, but also to limit possible negative effects (such as changed activity and/or instability of the protein kinase) caused by the protein kinase mutation itself. Nevertheless, the ASKA approach has been used in recent reports on HOG pathway control and target identification (Thorner \& Westfall 2006). In 2010, a selective inhibitor targeting the MAPK Hog1 was reported which undoubtedly will be a valuable tool investigating the HOG transduction pathway (Diner et al 2010).

\section{Microfluidic devices}

Depending on the size and application of the microfluidic devices, both the materials and developmental process used to manufacture the devices vary greatly. For biological applications the analyte is of course of crucial importance and different microfluidic designs and qualities are thus required if an organism, a cell, a metabolite or intracellular structure will be studied. In any case, the construction of the microfluidic device should be validated both theoretically and practically before use. Theoretical simulations of flows through the microfluidic channels are a valuable tool to evaluate new microfluidic designs and also provides optimum dimensions of the microfluidic device.

\subsection{Flow simulations}

The flows within microfluidic devices are usually laminar and characterised by a high surface area to volume ratio. This is an advantage when designing the system since it provides a possibility to beforehand estimate the flow profile at different locations within the device when changing the channel dimensions. The flows are expressed in terms of the Reynolds numbers (Re); a dimensionless number that depend on the fluid's density $(\rho)$,

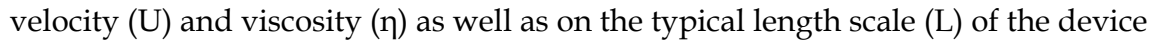

$$
\operatorname{Re}=\frac{\rho U L}{\eta}
$$

Generally, flows with a Reynolds number smaller than 2300 are considered laminar, while a larger Reynolds number represents flows of turbulence (Beebe et al 2002). When working with microfluidic devices, where at least one of the dimensions is on the microscale, the Reynolds numbers are usually well below 1 . Consequently, two adjacent fluids within a microfluidic device are mixed only by diffusion. The diffusion is driven by Brownian motions and is characterised by the diffusion coefficient, D. The diffusion coefficient is inversely proportional to the size of the diffusing particle, where the denominator is constituted by the so called Stokes drag coefficient

$$
D=\frac{k_{B} T}{6 \pi \eta \alpha}
$$

The Stokes drag coefficient involves the viscosity of the fluid ( $\eta$ ), and the radius of the particle (a), while the nominator contains Boltzmann's constant $\left(\mathrm{k}_{\mathrm{B}}\right)$ and the absolute temperature $(\mathrm{T})$. Even though several theoretical diffusion coefficients are available in the 
literature, specific substances used should if possible be experimentally measured by e.g. diffusion NMR. The effect of diffusion on a relevant time scale is of great importance on the microscale and the distance (d) a molecule diffuses over the time ( $\mathrm{t}$ ) can be calculated by

$$
d=\sqrt{2 D_{t}}
$$

Another important aspect is the flow profile of the fluids within the microfluidic device. Pressure driven forces, the geometry of the channels as well as surface tensions and surface to volume ratios affect the net flow through the device and need to be taken into consideration when simulated. Hence, theoretical simulations of specific substance within the flows are required for correct experimental setup. Figure 5 shows a simulation of 500 $\mathrm{mM}$ sorbitol through a three-channel microfludic chamber at a flow speed of $1000 \mathrm{nl} / \mathrm{min}$ while the flows in the two other channels are $0 \mathrm{nl} / \mathrm{min}$. This specific chamber is $27 \mu \mathrm{m}$ high, the wider inlet channel is $200 \mu \mathrm{m}$ wide, the thinner channels are $100 \mu \mathrm{m}$ wide and the outlet channel is $400 \mu \mathrm{m}$ wide.

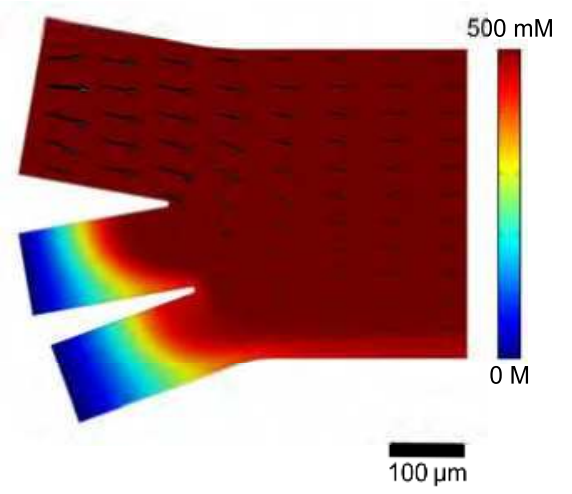

Fig. 5. Simulated concentration and flow profile at the surface of a three-channel microfluidic chamber during a static stress experiment. The lengths of the arrows are proportional to the flow velocities in the different inlet channels. The colours represent the concentration gradient of the perturbation agent sorbitol; blue corresponds to the lowest concentration of $0 \mathrm{mM}$ sorbitol and red the highest concentration $(500 \mathrm{mM}$ sorbitol). This simulation is valid for $1000 \mathrm{nl} / \mathrm{min}$ velocity flow in the sorbitol inlet channel and no flow in the lower two inlet channels.

\subsection{Construction of microfluidic devices}

Microfluidic devices can with advantage be constructed using a soft lithography approach. Usually a mould representing the microfluidic system is created, onto which a plastic polymer is added. After hardening, the plastic polymer will thus have the form of the wanted microfluidic device. The mould is made from a light sensitive photoresist that is dispensed on top of a planar silicon wafer. The liquid resist is spun out in a vacuum centrifuge to create a uniformly thick layer of photoresist. Different density properties of the resist will thus result in different layer thicknesses at specific centrifugation speeds. One commonly used resist is SU-8, which has good properties for achieving nearly vertical walls 
even when relatively thick resist layers are required. Typically, spin speed between 1000 and $3000 \mathrm{rpm}$ for approximately 30-50 seconds results in 1.5-250 $\mu \mathrm{m}$ thick resist layers, depending on which type of SU-8 is used. The SU-8 film needs to be densified and its solvents evaporated. This is done by heating steps; first at $65^{\circ} \mathrm{C}$ and a subsequent incubation at $95^{\circ} \mathrm{C}$. This gradual heating is recommended and the incubation times are closely related to the SU-8 thickness. A near UV-exposure is required to start the polymerisation process. This is preferably done in a so called mask aligner (i-line at $365 \mathrm{~nm}$ is recommended) where parallel rays from a UV light source illuminate the exposed surface of SU-8. To achieve selective cross-linkage of the resist, a post exposure bake step must be performed. Again, a stepwise heating (first at $65^{\circ} \mathrm{C}$ and then at $95^{\circ} \mathrm{C}$ ) is recommended to minimise wafer stress and/or cracking. Non-cross-linked resist is removed in a developer solution wash, for instance in ethyl lactate or diacetone alcohol, after which the master is rinsed with isopropanol and blown dry with nitrogen. Should the surface of the wafer contain a cloudy film after this treatment, a longer developer incubation time is necessary. There are both positive and negative photoresists available. A positive resist will become soluble in the developer after UV light illumination, while a negative photoresist will not. Hence, using a negative photoresist and a mask that corresponds to the microfluidic channel pattern will result in ridges of polymerised resist on top of the silicon wafer. This is referred to as the master and can also be reused several times. The inclusion of a final hard bake step at 150$200^{\circ} \mathrm{C}$ further increases the cross linking process and hence the lifetime of the master.

The most commonly used soft polymer in microfluidics today is polydimethylsiloxane (PDMS). This polymeric organo-silicon is considered to be inert and non-toxic, has optic transparency (lower limit of $280 \mathrm{~nm}$ ) and is rather inexpensive. Even though the surface is highly hydrophobic after curing, the surface properties can easily be modified (Whitesides et al 2000). The PDMS is constituted by repeats of the monomer $\left[\mathrm{SiO}\left(\mathrm{CH}_{3}\right)_{2}\right]$ that after addition of the curing agent $(1: 10 \mathrm{w} / \mathrm{w})$ is two-dimensionally cross-linked. When moulding the microfluidic system, PDMS is mixed with the curing agent and degassed in a vacuum dessicator. After approximately $10 \mathrm{~min}$ in the desiccators the vacuum is released and the PDMS mixture is dispensed on top of the master and incubated at $95^{\circ} \mathrm{C}$ for one hour to polymerise. Access holes to the microfluidic channels can either be drilled afterwards or made already during the moulding phase by the use of flattened needles centred to the ridges of the master. The polymerised PDMS can be peeled off and the master reused for a new moulding procedure. To tightly seal the PDMS system onto a glass slide, oxygen plasma treatment of the two is preferable. This can for instance be achieved in a plasma cleaner where only 30 seconds of air plasma treatment is necessary for the methyl groups in the PDMS to be replaced for hydroxyl groups $(-\mathrm{OH})$. When put in contact with the plasma treated glass, irreversible, covalent bonds (-Si-O-Si-) are spontaneously formed.

Figure 6 shows a thick PDMS device, connected via teflon tubing to small volume syringes with a total volume of $250 \mu$ that in turn can be placed in a syringe pump that pump the syringe fluids into the microfluidic device.

\section{The applications of microfluidics}

Microfluidics have complemented biological and biochemical research in many ways and the development of many variants of LOC and $\mu$ TAS indicates the vast variety of applications that could be performed (Chiu \& Kuo 2011; Cho et al 2010; Levchenko et al 


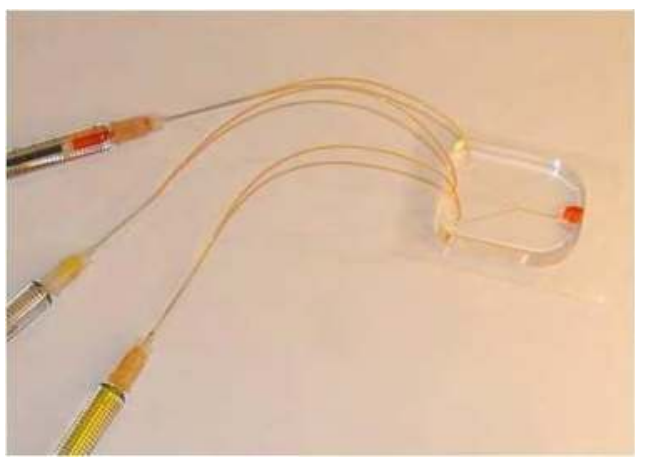

Fig. 6. A three-inlet microfluidic PDMS chamber. Syringes fitted to disposable needles and subsequent tubing attached. To visualise the channels the syringes contain highly concentrated fluorescein.

2010; Lu et al 2010; Park \& Hwang 2011; Veres et al 2010). However, cell biology would benefit even more if the level of control could be extended not only to include the microenvironment around the cells, but also the cells themselves. Optical manipulation and optical tweezers (OT) are optical techniques that provide the opportunity to manipulate the cells inside the microfluidic device in a non-invasive and non-intrusive manner.

The following part will give an example of how a microfluidic setup can be used in a single cell analysis project focusing on perturbations of the HOG MAPK pathway.

\subsection{The microscope and optical tweezers setup}

OT can easily be integrated into most optical microscopes setup and combined with a range of fluorescence imaging techniques. The setup used for the single cell experiments this chapter is based upon was built around an inverted epi-fluorescence microscope (Leica DMI6000B) containing a single stationary OT. In epi-fluorescence microscopy the microscope objective not only collects the emitted fluorescence light but also illuminates the sample with the excitation light. The equipment used for imaging the sample was a mercury metal halide bulb in combination with a filter cube containing excitation and emission filters for imaging green fluorescent protein (GFP) (excitation peak at $396 \mathrm{~nm}$ ) and mCherry (excitation peak at $587 \mathrm{~nm}$ ) as well as a dichroic mirror. The images were acquired by a 14 bit dynamic electron multiplying charged coupled device (EMCCD) that can detect very weak intensities emitted by the sample. The microscope was also equipped with a motorized stage and a motorized objective that allowed for optical sectioning of the sample.

The OT consisted of a laser beam $(\lambda=1070 \mathrm{~nm})$ with a Gaussian intensity profile that was focused to a diffraction limited beam waist by the same microscope objective that was used to image the sample. The laser beam was introduced into the microscope via a dichroic mirror that was situated between the microscope objective and the filter cube cassette. This enabled the imaging filters to be changed without affecting the laser trapping (Eriksson et al 2010). At the laser focus the power was estimated to be approximately $240 \mathrm{~mW}$ and allowed the trapping of yeast cells from one of the inlet channels within the microfluidic device. The theory behind the optical trapping mechanisms and important experimental 
considerations is beyond the scope of this chapter and can be found elsewhere (e.g. (Neuman \& Block 2004)).

\subsection{The imaging of the cells}

The development of the green fluorescent protein (GFP) has really come to revolutionise the field of biological imaging (Chalfie et al 1994). Since its discovery, GFP and other fluorescent proteins have been applied in a wide range of applications (Chudakov et al; Ckurshumova et al; Palmer et al 2011; Shaner et al 2007; Tsien et al 2005). By PCR, the gene sequence of the fluorescent protein can be inserted at almost any position in the gene of interest. The cells are either transiently or constitutively expressing one or several fluorescent protein-fusion proteins (i.e. reporter proteins), that can thus be monitored both spatially and temporally on a sub-cellular level. As a consequence, cellular events such as dynamic intracellular signalling, transport and localisation, protein-protein interactions and gene expression can easily be monitored using an ordinary fluorescence microscope.

When elucidating the mechanisms of the HOG transduction pathway, the cells are engineered to express GFP coupled Hog1 (Hog1-GFP). This enables the localisation of Hog1 and the protein can be imaged during the performed time lapse studies while the cell is perturbed by rapid environmental changes. Cells expressing the general stress protein Msn2 coupled to GFP (Msn2-GFP) were used as control in the experiments. Msn2 is also cytosolic during normal conditions, but migrates into the nucleus upon osmotic stress (Gorner et al 1998). Both cell types also express the nucleus resident reporter protein, Nrd1-mCherry, which facilitates cell nuclei localisation during imaging. The cells were imaged in 3D by seven optical sections (using the filter for GFP and mCherry) and followed for 45 minutes. Since the cellular response to the environmental change occurs within minutes following the perturbation, images was captured every 30 seconds in the beginning of the time lapse study. After approximately 5 minutes, the images were captured more and more sparsely (see Figure 11).

\subsection{Microfluidics in single cell analysis}

To improve cell adhesion, the microfluidic device is flushed with the lectin protein Concanavalin A (ConA). ConA specifically binds to the glucose aminoglycans residues on the cell wall of yeast cells, and distributed to the microfluidic device at a concentration of 1 $\mathrm{mg} / \mathrm{ml}$ for $30-60 \mathrm{~min}$ prior to experiment is enough to acquire proper cell binding to the surface (Eriksson et al 2010). The yeast cells as well as the other substances or mediums needed for the experiment are introduced into the microfluidic channels via tubing coupled to glass syringes. The syringes are in turn placed in syringe pumps that allow separate control of the flow speeds in each channel. When a stable flow from all inlet channels is established, the optical tweezers are used to trap single cells flowing past. In our threechannel chamber, this is done while the pump speed settings are $80 \mathrm{nl} / \mathrm{min}$ in the two thinner inlet channels and $40 \mathrm{nl} / \mathrm{min}$ in the wider one. In figure 7, yeast cells are introduced in the lower channel while the middle channel is "the neutral channel", containing the same solutions as the cells have been cultured in. The third, wider channel contains the perturbation solution. 


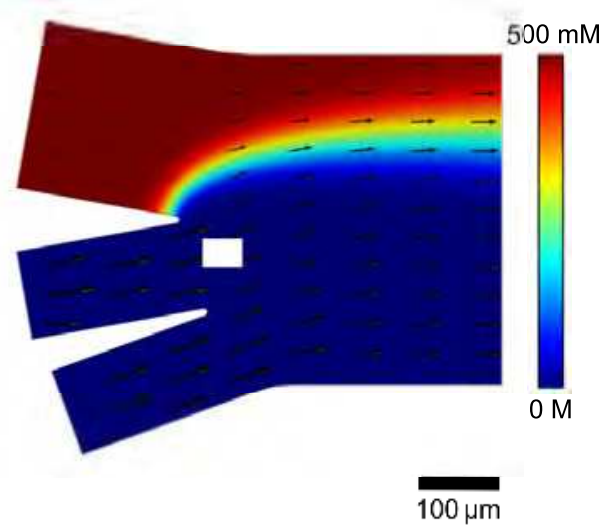

Fig. 7. Simulated concentration and flow profile at the surface of a three-channel microfluidic chamber during loading of the cell array using the OT. The lengths of the arrows are proportional to the flow velocity which is also printed in each of the inlet channels ( $\mathrm{nl} / \mathrm{min})$. The colours represent the concentration gradient of the perturbation agent sorbitol; blue corresponds to the lowest concentration of $0 \mathrm{mM}$ sorbitol and red the highest concentration ( $500 \mathrm{mM}$ sorbitol). This simulation is valid for $40 \mathrm{nl} / \mathrm{min}$ velocity flow in the sorbitol inlet channel and $80 \mathrm{nl} / \mathrm{min}$ velocity flow in the lower two inlet channels. The cells are introduced in the lower inlet channel and the white area shows where the cell array is placed.

The cells are individually trapped by the OT and attached to the bottom surface of the device. The trapping to the release of the cell into the array usually takes less than 5 seconds, which leave the cells reproduction ability unaffected by the laser light (Eriksson et al 2010). The effective area of the cell array depends both the width of the channels as well as on the diffusion coefficient of the substances used in the experiment. When using for instance the combination of a three-channel microfluidic device with sorbitol as the perturbing agent, up to 25 cells can be placed in the array that constitutes the area of interest. The environment of the cells attached to the bottom of the system is changed by changing the individual pump speeds. As visualised in figure 8 the 25 cells are placed within the area of the white rectangle in a three-channel chamber. Fluorescein is introduced in the upper channel to visualise the borders of fluids as would be the case during yeast cell trapping and placement. During this process, no fluorescein (i.e. the perturbation) reaches the cell array, leaving the cells unaffected (left panel). The environment is then changed by increasing the pump speed of the pump controlling the fluorescein flow while decreasing the pump speed controlling the other two flows (right panel). By using this procedure, a change in the environment can be successfully accomplished within 2 seconds. After the environmental switch the cell array is covered by the fluorescein and in the case of introducing a drug, the cellular response to the drug exposure can be imaged by the microscope. By automating and synchronising the pump speed and the image acquisition, the experimental precision can be increased. The removal of a substance or even multiple treatment pulses is thus possible by simply changing the speed of the individual flows within the device. 

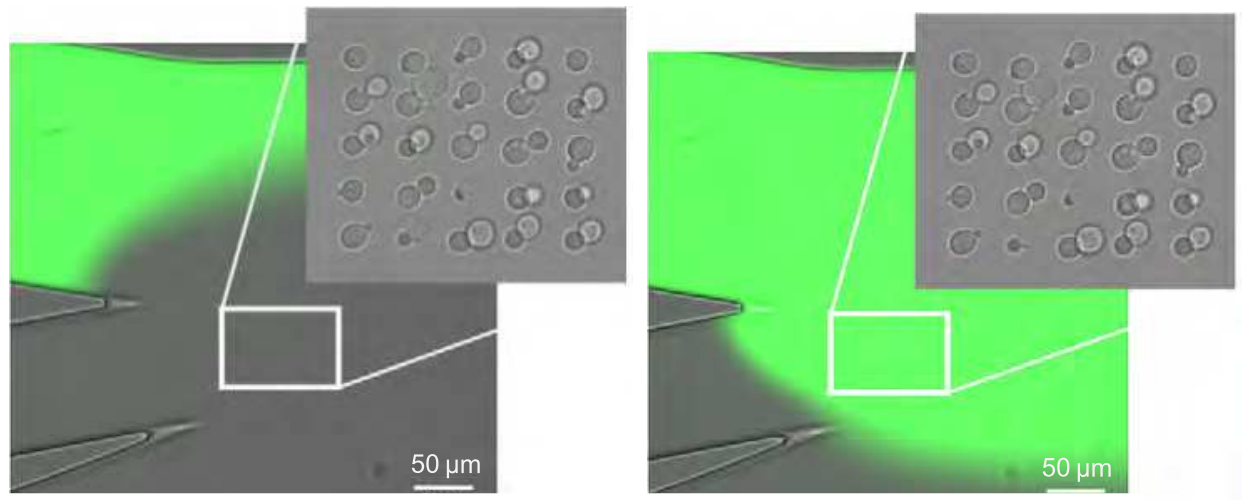

Fig. 8. Practical evaluations of the flow speeds in a three-inlet microfluidic chamber. The images show the field of view through a 20X objective. The upper inlet channel contains fluorescein and the white square corresponds to the placement of the yeast cell array (shown in enlargement with yeast cells in both images). The left image corresponds to the flow situations when capturing the cells by the OT and array placement. No perturbation (here represented by fluorescein) is affecting the cells. In the right image the pump speeds have been changed and the fluorescein now covers the cells in the array that consequently will be perturbed and monitored while responding. This environmental change is completed within 2 seconds.

A three-channel microfluidic chamber is a convenient setup for treating cells with one additional external stimulus. However, if yet another media or reagent needs to be introduced, a fourth channel would be required. This turned out to be necessary when the inhibitor against the MAPK Hog1 in the HOG pathway was to be evaluated. The inhibitor was designed to selectively bind to Hog1 and its effect on Hog1 nuclear migration upon osmotic stress treatment was thus to be investigated. There was, however, no other way of performing this experiment using a three-channel system but to pre-treat the cells with the inhibitor and then introduce the cells into the microfluidic device. Thus it was impossible to study cells that had been exposed to the inhibitor for less than $15 \mathrm{~min}$, since this was the minimum time from the inhibitor pre-treatment to the environmental change of the cells within the microfluidic device. It also turned out to be difficult to compare data from different experimental runs, since cells in different experiments had been exposed to the inhibition for different periods of time before the experiment could start. The four-channel microfluidic device shown in figure 9 enabled us not only to treat the yeast cells with the perturbing agent sorbitol, but also to perform the pre-treatment of the cells with the inhibitor for a predetermined time within the microfluidic device.

The workflow is as follows; (i) cells are optically trapped and placed in the array by OT. (ii) The cells are exposed to the inhibitor by increasing the flow speed in the inhibitor inlet channel, before (iii) the cells are perturbed by exposure to sorbitol by yet another speed change of the flow in the sorbitol inlet channel. Meanwhile the yeast cells were imaged and the Hog1 localisation monitored on a single cell basis. Another benefit of using an extra inlet channel was the possibility to also introduce control cells in the yeast cell array. The inhibitor was designed to bind to the ATP-binding site of Hog1 and hence inhibit 


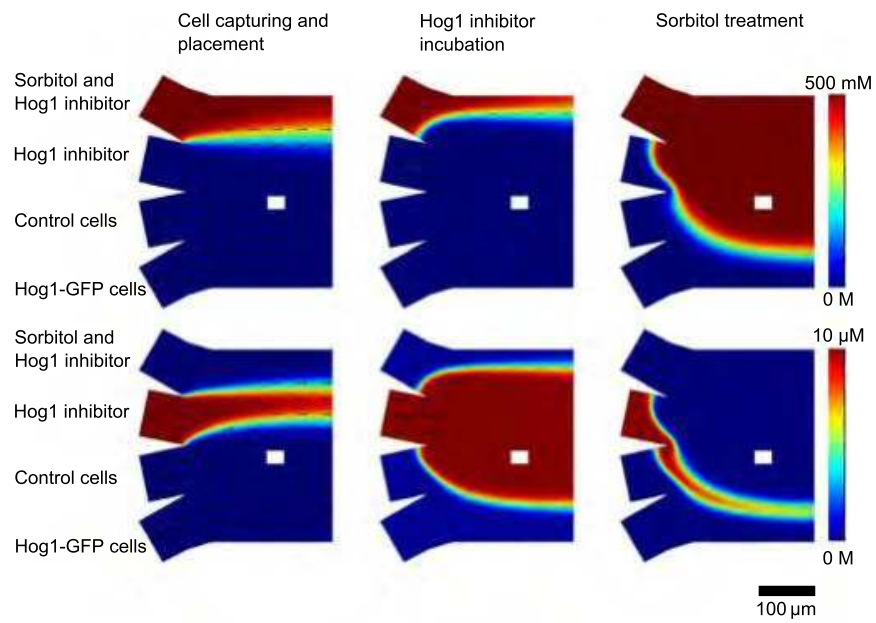

Fig. 9. Simulations of the flow profile used in the four-channel microfluidic chamber setup. In the upper channel, the perturbation of the MAPK pathway is introduced (sorbitol) in combination with the inhibitor; the second inlet channel from the top contains the inhibitor only; the third and fourth inlet channel from the top contains the control cells and Hog1GFP cells respectively. The three simulation scenarios (from left to right) represent cell capturing and placement, Hog1 inhibitor incubation and sorbitol treatment. The red colour in the upper row simulations correspond to $500 \mathrm{mM}$ sorbitol, while in the lower row simulations red colour corresponds to $10 \mu \mathrm{M}$ Hog1 inhibitor.

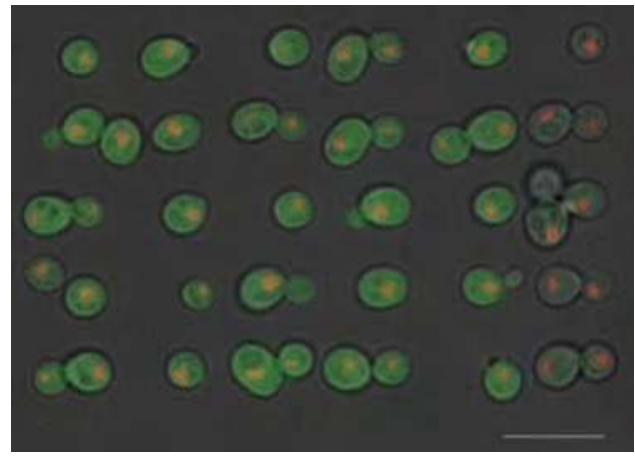

Fig. 10. Imaged response of the yeast cell array in the four-channel microfluidic chamber. The image shows the cell array at $t=-30$ seconds, i.e. before the perturbation is introduced. The last column of cells constitutes the control cells whose expressed reporter protein (Msn2-GFP) migration is not affected by the Hog1 inhibitor. All cells express a nuclear resident reporter protein (Nrd1-mCherry) that enables visual localisation of the nuclei at all times. The scale bar represents $10 \mu \mathrm{m}$.

downstream phosphorylation events in the HOG signal transduction pathway. Since it is suggested that the ATP-binding site of Hog1 must be active for nuclear envelope translocation (Thorner \& Westfall 2006), a complete and proper inhibitor-binding to Hog1 
would also inhibit Hog1 nuclear migration. Therefore, instead of imaging a "non-response" of the Hog1-inhibitor treated cells only, also cells expressing a reporter protein unaffected by the inhibitor but responsive to the osmotic stress was introduced. Such cells would also constitute a propriety control of the microfluidic chamber itself, ensuring that all the cells in the array indeed experience the osmotic stress (Figure 10). The cell response due to osmotic stress can be presented as the ratio (R) of nuclear to cytosolic Hog1-GFP and thus corresponds to the GFP intensity in the nucleus and cytosol respectively.

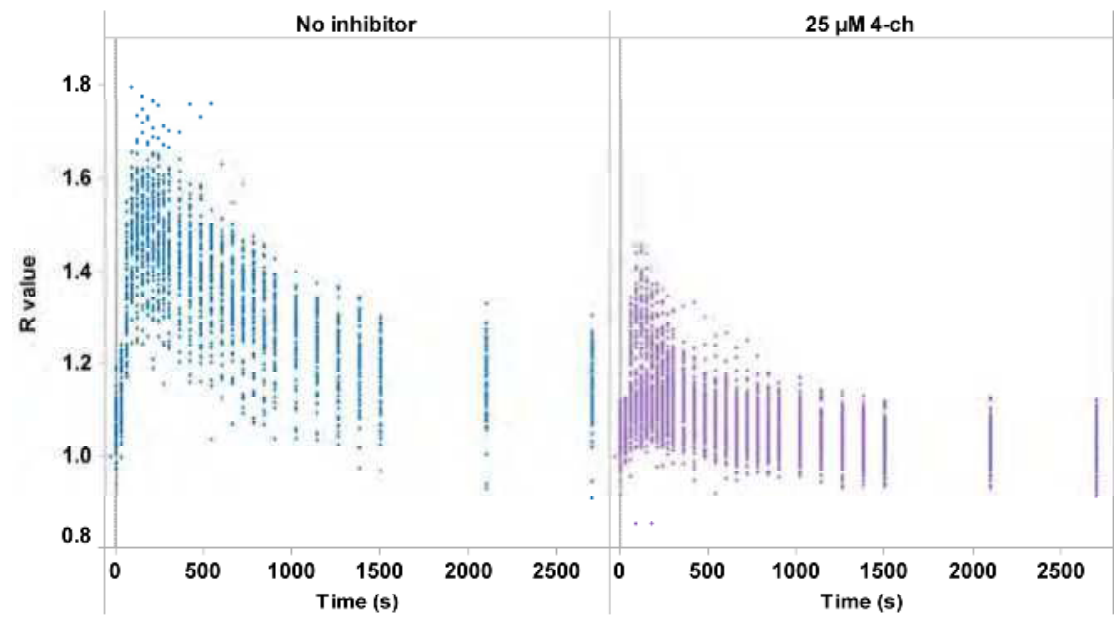

Fig. 11. Single cell data of the Hog1-GFP intensities. The left panel shows the normalised Rvalues of yeast cells experiencing sorbitol perturbation only (two separate experiments). The right panel shows the yeast cells that have been pre-treated with the Hog1 inhibitor for 20 minutes before sorbitol perturbation in the four-channel microfluidic chamber (four separate experiments). The sorbitol perturbation is initiated at time $=0$.

A high R-value is therefore attained during sorbitol perturbation where Hog1 has migrated into the nuclei. Pre-treating the cells with the Hog1 inhibitor would therefore impair the Rvalue increase upon sorbitol perturbation compared with cells experiencing sorbitol perturbation only. When treating the yeast cell array with $25 \mu \mathrm{M}$ of the Hog1 inhibitor for 20 minutes before sorbitol perturbation, the R-value did indeed decrease compared with cells that had experienced sorbitol perturbation only, as can be seen in figure 11. The graphs show the time lapse single cell data of two (no inhibitor treatment) and four ( $25 \mu \mathrm{M}$ Hog1 inhibitor treatment) separate experiments respectively. The Msn2-GFP in the control cells did migrate into the nucleus upon sorbitol perturbation and hence proved that sorbitol perturbation was indeed induced (data not shown).

\section{Conclusions and future aspects}

The experimental setup and the results obtained indicate how mechanisms of the MAPK HOG pathway can be investigated using a microfluidic approach. The four-channel microfluidic chamber demonstrated allows the removal of not only the sorbitol perturbation, but also the inhibitor, thus enabling inhibitor turnover rate investigations. The 
controlled exposure to stress substances as well as inhibitors allows kinetic and dynamic events to be studied with great temporal and spatial resolution, something that usually is very difficult to achieve with traditional techniques. The single cell analysis is also an excellent complement to ensemble averaging techniques, since the cellular heterogeneity within the genetically identical population is easily revealed. We are confident that similar microfluidic approaches will be a valuable tool in the future when delineating, not only the HOG pathway, but similar pathways in which dynamics of fluorescent reporter proteins can be followed in single cells. Furthermore, the inclusion of two different cell types in the same cell array enables a direct comparison between, for instance, cells with different reporter proteins or mutants versus wild type cells.

\section{Acknowledgments}

The authors thank Charlotte Hamngren for the development and evaluation of the fourchannel microfluidic chamber and Morten Grötli for the Hog1 inhibitor. The project was funded by the FP7 programme UNICELLSYS.

\section{References}

Ammerer G, Reiser V, Salah SM. 2000. Polarized localization of yeast Pbs2 depends on osmostress, the membrane protein Sho1 and Cdc42. Nat Cell Biol 2:620-7

Bayne ML, Applebaum J, Chicchi GG, Hayes NS, Green BG, Cascieri MA. 1988. Expression, Purification and Characterization of Recombinant Human Insulin-Like Growth Factor-I in Yeast. Gene 66:235-44

Beebe DJ, Mensing GA, Walker GM. 2002. Physics and applications of microfluidics in biology. Annu Rev Biomed Eng 4:261-86

Beebe DJ, Paguirigan AL. 2008. Microfluidics meet cell biology: bridging the gap by validation and application of microscale techniques for cell biological assays. Bioessays 30:811-21

Beggs JD. 1978. Transformation of Yeast by a Replicating Hybrid Plasmid. Nature 275:104-9

Bennett MR, Pang WL, Ostroff NA, Baumgartner BL, Nayak S, et al. 2008. Metabolic gene regulation in a dynamically changing environment. Nature 454:1119-22

Burgess JCMaSM. 2003. Yeast as a model organism. In Encyclopedia of Life Sciences

Chalfie M, Tu Y, Euskirchen G, Ward WW, Prasher DC. 1994. Green Fluorescent Protein as a Marker for Gene-Expression. Science 263:802-5

Chiu DT, Kuo JS. 2011. Disposable microfluidic substrates: Transitioning from the research laboratory into the clinic. Lab on a Chip 11:2656-65

Cho YK, Gorkin R, Park J, Siegrist J, Amasia M, et al. 2010. Centrifugal microfluidics for biomedical applications. Lab on a Chip 10:1758-73

Chudakov DM, Matz MV, Lukyanov S, Lukyanov KA. Fluorescent proteins and their applications in imaging living cells and tissues. Physiol Rev 90:1103-63

Ckurshumova W, Caragea AE, Goldstein RS, Berleth T. Glow in the Dark: Fluorescent Proteins as Cell and Tissue-Specific Markers in Plants. Mol Plant

Dean PN, Hoffman RA. 2007. Overview of flow cytometry instrumentation. Curr Protoc Cytom Chapter 1:Unit1

Deptala A, Bedner E, Darzynkiewicz Z. 2001. Unique analytical capabilities of laser scanning cytometry (LSC) that complement flow cytometry. Folia Histochem Cyto 39:87-9 
Dihazi H, Kessler R, Eschrich K. 2004. High osmolarity glycerol (HOG) pathway-induced phosphorylation and activation of 6-phosphofructo-2-kinase are essential for glycerol accumulation and yeast cell proliferation under hyperosmotic stress. J Biol Chem 279:23961-8

Diner P, Veide Vilg J, Kjellen J, Migdal I, Andersson T, et al. Design, synthesis, and characterization of a highly effective Hog1 inhibitor: a powerful tool for analyzing MAP kinase signaling in yeast. PLoS One 6:e20012

Eriksson E, Sott K, Lundqvist F, Sveningsson M, Scrimgeour J, et al. 2010. A microfluidic device for reversible environmental changes around single cells using optical tweezers for cell selection and positioning. Lab on a Chip 10:617-25

Ferreira C, van Voorst F, Martins A, Neves L, Oliveira R, et al. 2005. A member of the sugar transporter family, Stl1p is the glycerol/H+ symporter in Saccharomyces cerevisiae. Mol Biol Cell 16:2068-76

Goffeau A, Barrell BG, Bussey H, Davis RW, Dujon B, et al. 1996. Life with 6000 genes. Science 274:546-\&

Gorner W, Durchschlag E, Martinez-Pastor MT, Estruch F, Ammerer G, et al. 1998. Nuclear localization of the $\mathrm{C} 2 \mathrm{H} 2$ zinc finger protein $\mathrm{Msn} 2 \mathrm{p}$ is regulated by stress and protein kinase A activity. Genes Dev 12:586-97

Gustin MC, Albertyn J, Alexander M, Davenport K. 1998. MAP kinase pathways in the yeast Saccharomyces cerevisiae. Microbiol Mol Biol Rev 62:1264-300

Hartwell LH. 2004. Yeast and cancer. Bioscience Rep 24:523-44

Hinnen A, Hicks JB, Fink GR. 1978. Transformation of Yeast. P Natl Acad Sci USA 75:1929-33

Hohmann S. 2009. Control of high osmolarity signalling in the yeast Saccharomyces cerevisiae. Febs Lett 583:4025-9

Jensen KF, El-Ali J, Sorger PK. 2006. Cells on chips. Nature 442:403-11

Johnson LN, Noble MEM, Endicott JA. 2004. Protein kinase inhibitors: Insights into drug design from structure. Science 303:1800-5

Kitano H. 2002. Computational systems biology. Nature 420:206-10

Klis FM, Boorsma A, De Groot PW. 2006. Cell wall construction in Saccharomyces cerevisiae. Yeast 23:185-202

Levchenko A, Gupta K, Kim DH, Ellison D, Smith C, et al. 2010. Lab-on-a-chip devices as an emerging platform for stem cell biology. Lab on a Chip 10:2019-31

Lu H, Crane MM, Chung K, Stirman J. 2010. Microfluidics-enabled phenotyping, imaging, and screening of multicellular organisms. Lab on a Chip 10:1509-17

Maeda T, Takekawa M, Saito H. 1995. Activation of Yeast Pbs2 Mapkk by Mapkkks or by Binding of an Sh3-Containing Osmosensor. Science 269:554-8

Manz A, Auroux PA, Iossifidis D, Reyes DR. 2002a. Micro total analysis systems. 2. Analytical standard operations and applications. Analytical Chemistry 74:2637-52

Manz A, Reyes DR, Iossifidis D, Auroux PA. 2002b. Micro total analysis systems. 1. Introduction, theory, and technology. Analytical Chemistry 74:2623-36

Mcaleer WJ, Buynak EB, Maigetter RZ, Wampler DE, Miller WJ, Hilleman MR. 1984. Human Hepatitis-B Vaccine from Recombinant Yeast. Nature 307:178-80

Molina M, Martin H, Flandez M, Nombela C. 2005. Protein phosphatases in MAPK signalling: we keep learning from yeast. Mol Microbiol 58:6-16

Mortimer RK, Johnston JR. 1959. Life Span of Individual Yeast Cells. Nature 183:1751-2

Neuman KC, Block SM. 2004. Optical trapping. Rev Sci Instrum 75:2787-809 
O'Rourke SM, Herskowitz I. 2004. Unique and redundant roles for HOG MAPK pathway components as revealed by whole-genome expression analysis. Mol Biol Cell 15:53242

O'Shea EK, Raser JM. 2004. Control of stochasticity in eukaryotic gene expression. Science 304:1811-4

Ono K, Han J. 2000. The p38 signal transduction pathway: activation and function. Cell Signal 12:1-13

Palmer AE, Qin Y, Park JG, McCombs JE. 2011. Design and application of genetically encoded biosensors. Trends Biotechnol 29:144-52

Park JK, Hwang H. 2011. Optoelectrofluidic platforms for chemistry and biology. Lab on a Chip 11:33-47

Pepperkok R, Ellenberg J. 2006. Innovation - High-throughput fluorescence microscopy for systems biology. Nat Rev Mol Cell Bio 7:690-6

Ploger R, Zhang J, Bassett D, Reeves R, Hieter P, et al. 2000. XREFdb: cross-referencing the genetics and genes of mammals and model organisms. Nucleic Acids Res 28:120-2

Posas F, WurglerMurphy SM, Maeda T, Witten EA, Thai TC, Saito H. 1996. Yeast HOG1 MAP kinase cascade is regulated by a multistep phosphorelay mechanism in the SLN1-YPD1-SSK1 "two-component" osmosensor. Cell 86:865-75

Rep M, Krantz M, Thevelein JM, Hohmann S. 2000. The transcriptional response of Saccharomyces cerevisiae to osmotic shock. Hot1p and Msn2p/Msn4p are required for the induction of subsets of high osmolarity glycerol pathway-dependent genes. J Biol Chem 275:8290-300

Rep M, Reiser V, Gartner U, Thevelein JM, Hohmann S, et al. 1999. Osmotic stress-induced gene expression in Saccharomyces cerevisiae requires Msn1p and the novel nuclear factor Hot1p. Mol Cell Biol 19:5474-85

Ryley J, Pereira-Smith OM. 2006. Microfluidics device for single cell gene expression analysis in Saccharomyces cerevisiae. Yeast 23:1065-73

Saito H, Posas F. 1998. Activation of the yeast SSK2 MAP kinase kinase kinase by the SSK1 two-component response regulator. Embo J 17:1385-94

Shaner NC, Patterson GH, Davidson MW. 2007. Advances in fluorescent protein technology. J Cell Sci 120:4247-60

Shields DC, Caffrey DR, O'Neill LAJ. 1999. The evolution of the MAP kinase pathways: Coduplication of interacting proteins leads to new signaling cascades. J Mol Evol 49:567-82

Shokat KM, Bishop AC, Ubersax JA, Petsch DT, Matheos DP, et al. 2000. A chemical switch for inhibitor-sensitive alleles of any protein kinase. Nature 407:395-401

Silver PA, Ferrigno P, Posas F, Koepp D, Saito H. 1998. Regulated nucleo/cytoplasmic exchange of HOG1 MAPK requires the importin beta homologs NMD5 and XPO1. Embo J 17:5606-14

Specht KM, Shokat KM. 2002. The emerging power of chemical genetics. Curr Opin Cell Biol 14:155-9

Stagljar I, Suter B, Auerbach D. 2006. Yeast-based functional genomics and proteomics technologies: the first 15 years and beyond. Biotechniques 40:625-44

Tamas MJ, Thorsen M, Di YJ, Tangemo C, Morillas M, et al. 2006. The MAPK Hog1p modulates Fps1p-dependent arsenite uptake and tolerance in yeast. Mol Biol Cell 17:4400-10 
Tatebayashi K, Tanaka K, Yang HY, Yamamoto K, Matsushita Y, et al. 2007. Transmembrane mucins Hkr1 and Msb2 are putative osmosensors in the SHO1 branch of yeast HOG pathway. Embo J 26:3521-33

Thorner J, Westfall PJ. 2006. Analysis of mitogen-activated protein kinase signaling specificity in response to hyperosmotic stress: Use of an analog-sensitive HOG1 allele. Eukaryot Cell 5:1215-28

Thorner J, Westfall PJ, Ballon DR. 2004. When the stress of your environment makes you go HOG wild. Science 306:1511-2

Thorner J, Westfall PJ, Patterson JC, Chen RE. 2008. Stress resistance and signal fidelity independent of nuclear MAPK function. P Natl Acad Sci USA 105:12212-7

Tsien RY, Shaner NC, Steinbach PA. 2005. A guide to choosing fluorescent proteins. Nat Methods 2:905-9

Tudos AJ, Besselink GAJ, Schasfoort RBM. 2001. Trends in miniaturized total analysis systems for point-of-care testing in clinical chemistry. Lab on a Chip 1:83-95

van den Berg A, Andersson H. 2003. Microfluidic devices for cellomics: a review. Sensor Actuat B-Chem 92:315-25

van den Berg A, Lammerink TSJ. 1998. Micro total analysis systems: Microfluidic aspects, integration concept and applications. Top Curr Chem 194:21-49

Warmka J, Hanneman J, Lee J, Amin D, Ota I. 2001. Ptc1, a type 2C Ser/Thr phosphatase, inactivates the HOG pathway by dephosphorylating the mitogen-activated protein kinase Hog1. Mol Cell Biol 21:51-60

Weibel DB, Diluzio WR, Whitesides GM. 2007. Microfabrication meets microbiology. Nat Rev Microbiol 5:209-18

Veres T, Malic L, Brassard D, Tabrizian M. 2010. Integration and detection of biochemical assays in digital microfluidic LOC devices. Lab on a Chip 10:418-31

Whitesides GM. 2006. The origins and the future of microfluidics. Nature 442:368-73

Whitesides GM, McDonald JC, Duffy DC, Anderson JR, Chiu DT, et al. 2000. Fabrication of microfluidic systems in poly(dimethylsiloxane). Electrophoresis 21:27-40

Whitesides GM, Sia SK. 2003. Microfluidic devices fabricated in poly(dimethylsiloxane) for biological studies. Electrophoresis 24:3563-76 


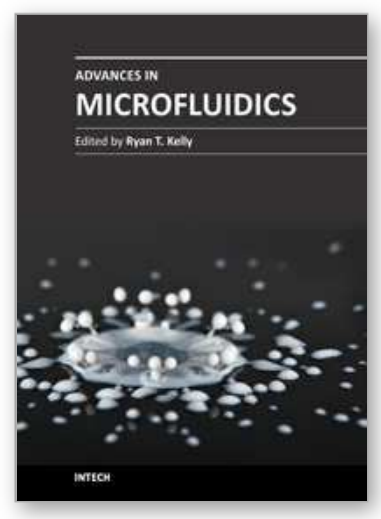

\author{
Advances in Microfluidics \\ Edited by Dr. Ryan Kelly
}

ISBN 978-953-51-0106-2

Hard cover, 250 pages

Publisher InTech

Published online 07, March, 2012

Published in print edition March, 2012

Advances in Microfluidics provides a current snapshot of the field of microfluidics as it relates to a variety of sub-disciplines. The chapters have been divided into three sections: Fluid Dynamics, Technology, and Applications, although a number of the chapters contain aspects that make them applicable to more than one section. It is hoped that this book will serve as a useful resource for recent entrants to the field as well as for established practitioners.

\title{
How to reference
}

In order to correctly reference this scholarly work, feel free to copy and paste the following:

Caroline Beck and Mattias Goksör (2012). Microfluidics in Single Cell Analysis, Advances in Microfluidics, Dr. Ryan Kelly (Ed.), ISBN: 978-953-51-0106-2, InTech, Available from:

http://www.intechopen.com/books/advances-in-microfluidics/microfluidics-in-single-cell-analysis

\section{INTECH}

open science | open minds

\author{
InTech Europe \\ University Campus STeP Ri \\ Slavka Krautzeka 83/A \\ 51000 Rijeka, Croatia \\ Phone: +385 (51) 770447 \\ Fax: +385 (51) 686166 \\ www.intechopen.com
}

\author{
InTech China \\ Unit 405, Office Block, Hotel Equatorial Shanghai \\ No.65, Yan An Road (West), Shanghai, 200040, China \\ 中国上海市延安西路65号上海国际贵都大饭店办公楼 405 单元 \\ Phone: +86-21-62489820 \\ Fax: $+86-21-62489821$
}


(C) 2012 The Author(s). Licensee IntechOpen. This is an open access article distributed under the terms of the Creative Commons Attribution 3.0 License, which permits unrestricted use, distribution, and reproduction in any medium, provided the original work is properly cited. 